\title{
EphA2 receptor activation with ephrin-A1 ligand restores cetuximab efficacy in $N R A S$-mutant colorectal cancer cells
}

\author{
ELISABET CUYÀS ${ }^{1,2^{*}}$, BERNARDO QUERALT ${ }^{3,4^{*}}$, BEGOÑA MARTIN-CASTILLO ${ }^{2,5}$, \\ JOAQUIM BOSCH-BARRERA ${ }^{3,4}$ and JAVIER A. MENENDEZ ${ }^{1,2}$ \\ ${ }^{1}$ ProCURE (Program Against Cancer Therapeutic Resistance), Metabolism and Cancer Group, \\ Catalan Institute of Oncology; ${ }^{2}$ Girona Biomedical Research Institute (IDIBGI); \\ ${ }^{3}$ Department of Medical Oncology, Catalan Institute of Oncology; \\ ${ }^{4}$ Department of Medical Sciences, Medical School, University of Girona; \\ ${ }^{5}$ Unit of Clinical Research, Catalan Institute of Oncology, Girona, Catalonia, Spain
}

Received February 20, 2017; Accepted May 8, 2017

DOI: $10.3892 / o r .2017 .5682$

\begin{abstract}
Patients with wild-type KRAS metastatic colorectal cancer (mCRC) that harbors NRAS activating mutations do not benefit from anti-EGFR therapies. Very little is known about oncogenic NRAS signaling driving mCRC unresponsiveness to the EGFR-directed antibody cetuximab. Using a system of paired NRAS-mutant and wild-type isogenic mCRC cell lines to explore signaling pathways engaged by the common oncogenic NRAS Q61K variant upon challenge with cetuximab, we uncovered an unexpected mechanism of resistance to cetuximab involving dysregulation of the ephrin-A1/EphA2 signaling axis. Parental $N R A S^{+/+}$cells, but not $N R A S^{Q 61 K /+}$ cells, activated the ephrin receptor ephA1 in response to cetuximab treatment. Moreover, whereas cetuximab treatment significantly downregulated EPHA2 gene expression in $N R A S^{+/+}$ cells, EPHA2 expression in NRAS $S^{Q 61 K /+}$ cells was refractory to cetuximab. Remarkably, pharmacologically mimicked ephrin-A1 engagement to ephA 2 converted NRAS-mutant into $R A S$ wild-type mCRC cells in terms of cetuximab efficacy. Accordingly, activation of the ephA2 receptor by bioactive recombinant human ephrin-A1/Fc-fusion protein suppressed the cetuximab-unresponsive hyperactivation of MAPK and AKT and fully restored cetuximab activity in NRAS-mutant colorectal cells. Collectively, these findings reveal that the clinical benefit of cetuximab in mCRC might necessarily involve the suppression of the ligandless oncogenic signaling of the ephA2 receptor. Hence, ligand-dependent tumor
\end{abstract}

Correspondence to: Dr Javier A. Menendez, Girona Biomedical Research Institute (IDIBGI), Edifici M2, Parc Hospitalari Martí i Julià, E-17190 Salt, Girona, Spain

E-mail: jmenendez@iconcologia.net; jmenendez@idibgi.org

${ }^{*}$ Contributed equally

Key words: colon cancer, KRAS, NRAS, cetuximab, ephrins suppressor signaling using therapeutic ephA2 agonists might offer new therapeutic opportunities to clinically widen the use of cetuximab in NRAS-mutated and/or ephA2-dependent mCRC tumors.

\section{Introduction}

NRAS mutations occur in $\sim 3-5 \%$ of metastatic colorectal carcinoma (mCRC) patients and have been associated with lower disease control and response rates to the epidermal growth factor receptor (EGFR)-targeted monoclonal antibody cetuximab (1-8). Because patients with wild-type $K R A S$ mCRC that harbor NRAS activating mutations do not derive benefit from the administration of cetuximab, all major international clinical guidelines recommend restricting its use to mCRC patients with wild-type $R A S$ tumors $(6,9,10)$. Although previous data indicated that NRAS might provide similar or identical oncogenic signals to those of $K R A S$, as they are not typically found in the same tumor $(11,12)$, accumulating evidence suggests very distinct clinical consequences for the mutually exclusive $K R A S$ - and $N R A S$-mutant mCRC subsets $(13,14)$. Dissimilar biological consequences for mutations of KRAS and NRAS, which appear to be selected under distinct tumorigenic contexts, underlie their clinical distinction in mCRC patients. Accordingly, NRAS mutations, which appear to arise specifically under settings of continuous exposure to apoptotic stimuli in the context of chronic inflammation, provide a MAPK-related distinct, prosurvival signaling environment that mutational activation of KRAS does not (14).

An important unresolved question arising from the above observation is whether the apparently unique phenotype of mutant $N R A S$ can be exploited as a therapeutic strategy to circumvent the refractoriness to cetuximab. Whereas most studies have focused on investigating the downstream effectors of KRAS signaling for bypassing the response of $K R A S$-mutant mCRC cells to anti-EGFR therapy, almost nothing is known about the specific pathways employed by $N R A S$-mutant mCRC cells that render them unresponsive to 
cetuximab. Here we used isogenic $\mathrm{mCRC}$ cell lines to explore signaling pathways specifically engaged by the most common oncogenic NRAS Q61K variant upon challenge of mCRC cells with cetuximab. We provide evidence for an unexpected deregulation of the erythropoietin-producing hepatocellular (Eph) receptor tyrosine kinase (RTK)/ephrin ligand cell communication system (EphA2/ephrin-A1), which negatively influences cetuximab efficacy in NRAS-mutant mCRC cells.

\section{Materials and methods}

Cell lines. The X-MAN ${ }^{\mathrm{TM}}$ isogenic cell lines SW48 NRAS-WT $\left(N R A S^{+/+}\right.$) and SW48 NRAS $S^{Q 61 K /+}$ (cat no. HD 103-017), were purchased from Horizon Discovery Ltd. (Cambridge, UK) and maintained following the manufacturer's instructions in RPMI-1640 medium with $2 \mathrm{mmol} / \mathrm{l} \mathrm{L}$-glutamine, $25 \mathrm{mmol} / 1$ sodium bicarbonate and $10 \%$ fetal bovine serum.

Drugs and materials. Cetuximab was provided by the Hospital Universitari de Girona Dr Josep Trueta Pharmacy. Bioactive recombinant human EphrinA1/Fc (EA1-Fc; cat no. 6417-A1) was purchased from R\&D Systems (Minneapolis, MN, USA) and dissolved in PBS.

Cell proliferation. Cells were plated in 24-well plates at 5,000 cells/well and incubated for $18 \mathrm{~h}$ in a humidified atmosphere containing $5 \%$ carbon dioxide at $37^{\circ} \mathrm{C}$ to allow for attachment, after which a zero-time point was determined. Cells were grown in regular medium with or without $100 \mu \mathrm{g} / \mathrm{ml}$ cetuximab and counted with a Coulter Counter (Coulter Electronics Inc., Hialeah, FL, USA). All assays were performed at least twice in triplicate.

Phospho-proteome profiling. Phospho-receptor screening was performed using Proteome Profiler Human Phospho-RTK array (R\&D Systems) according to the manufacturer's instructions. Densitometry analyses of the scanned phospho-arrays were carried out using Carestream Molecular Imaging Software (Carestream Health, Rochester, NY, USA).

RNA isolation and reverse transcription. Total RNA was extracted from cells using Nucleospin RNA plus kit (Macherey-Nagel GmbH \& Co. KG) according to the manufacturer's instructions. Two micrograms of total RNA was reverse-transcribed into cDNA using High Capacity cDNA Reverse Transcription kit (Thermo Fisher Scientific, Carlsbad, CA, USA) according to the manufacturer's instructions. RNA concentration and quality were determined in an ND-1000 spectrophotometer (NanoDrop ${ }^{\mathrm{TM}}$ ND-1000, NanoDrop Technologies, USA)

Gene expression. cDNA (50 ng) were assayed in triplicate according to established protocols using a QuantStudio ${ }^{\mathrm{TM}} 7$ Flex Real-Time PCR system (Thermo Fisher Scientific) with an automated baseline and threshold cycle detection. GAPDH and ACTB were used as reference genes. Primers and fluorescent probes for EPHA1, EPHA2, EFNA1, EFNA2, GAPDH, and ACTB were obtained from Thermo Fisher Scientific (TaqMan Gene Expression assays: assay ID Hs00358886-m1, Hs01023290_m1, Hs00178313_m1, Hs00171656-m,
Hs99999902_m1, and Hs99999903_m1, respectively). Data were analyzed using the Thermo Fisher Cloud software (Thermo Fisher Scientific).

Real-time cell growth rates. Proliferation was monitored in real time using the xCELLigence RTCA DP Instrument (ACEA Biosciences, San Diego, CA, USA). Cellular growth rate was determined by the slope of the growth curve using the RTCA Software Package 1.2. We conducted the normalization at one-time point before the treatment.

PathScan sandwich immunoassay. The PathScan ${ }^{\circledR}$ Intracellular Signaling array kit (cat no. 7323; Cell Signaling Technology, Danvers, MA, USA) was used as per the manufacturer's instructions.

Statistical analysis. Data are presented as mean \pm SD from at least three independent experiments. Two-group comparisons were performed using Student's t-test. Comparisons of means of $\geq 3$ groups were performed by ANOVA, and the existence of individual differences tested by Scheffé's multiple contrasts. P-values $<0.01$ were considered to be statistically significant. All statistical tests were two-sided.

\section{Results}

Heterozygous knock-in of the NRAS activating mutation Q61K is sufficient to allow escape from cetuximab-induced cell growth inhibition. We used an in vitro mCRC model of isogenic pairs of SW48 colon cancer cell lines in which one allele of the endogenous $N R A S$ gene contained a heterozygous knock-in of the c.181C $>$ A activating mutation resulting in an amino acid substitution from glutamine $(\mathrm{Q})$ to lysine $(\mathrm{K})$ at position $61\left(N R A S^{Q 61 K /+}\right)$. We previously reported that, whereas a strong reduction of cell viability was noted for parental $N R A S^{+/+}$cells cultured in the presence of $100 \mu \mathrm{g} / \mathrm{ml}$ cetuximab, $N R A S^{Q 61 K /+}$ cells were fully refractory to cetuximab-induced cell viability (15).

NRAS $S^{Q 61 K /+}$ cells fail to activate EphAl receptor tyrosine kinase in response to cetuximab. We first examined the changes in the phospho-proteome of isogenic $N R A S^{+/+}$and $N R A S^{Q 61 K /+}$ cells using the commercially available Proteome Profiler Human Phospho-RTK array kit. Phospho-RTK profiling revealed that the SW48-based model of mCRC mostly depends on EGFR signaling to proliferate since EGFR (HER1) was the tyrosine kinase receptor more significantly active among the 42 different phospho-receptor tyrosine kinases included in the array (Fig. 1). Treatment of $N R A S^{+/+}$and $N R A S^{Q 61 K /+}$ with cetuximab was found to further enhance the phosphorylation/ activation status of EGFR (Fig. 1), a phenomenon that was likely due to cetuximab-induced EGFR homodimerization and autophosphorylation as previously reported in non-smallcell lung cancer cells, head and neck squamous carcinoma cells, and triple-negative breast cancer cells (16-18).

Closer inspection of the relative levels of tyrosine phosphorylation detected by the array indicated that $N R A S^{+/+}$cells, albeit modestly, activated the endogenous ephrin receptor ephA1 in response to cetuximab, and this was more obvious with a longer exposure of the membrane. By contrast, ephA1 

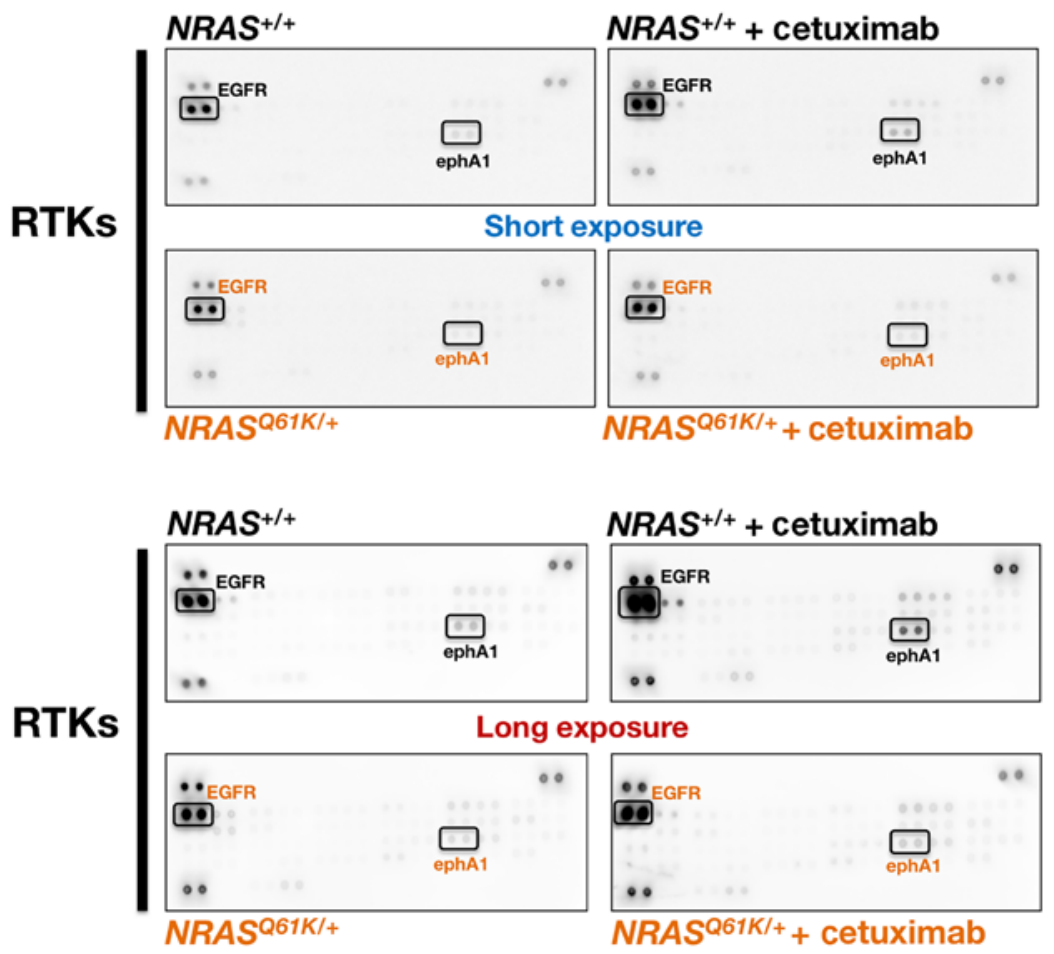

Figure 1. Cetuximab-induced activation of the EphA1 receptor is unresponsive in $N R A S$-mutant cells. Phospho-proteome profiling of mCRC cells in response to cetuximab. Total cell lysates $(750 \mu \mathrm{g})$ from $N R A S^{+/+}$and $N R A S^{Q 61 K /+}$ cells before and after treatment with $100 \mu \mathrm{g} / \mathrm{ml}$ cetuximab $(48 \mathrm{~h})$ were incubated on membranes of the phospho-proteomics platform as described in Materials and methods. Representative phospho-proteome analyses are shown. Equivalent results were obtained in two independent experiments.

was not activated in cetuximab-refractory $N R A S^{Q 61 K /+}$ cells in response to cetuximab (Fig. 1).

Cetuximab fails to downregulate EphA2 in NRAS-mutant $m C R C$ cells. The EPH gene family is the largest subfamily of RTKs, including at least 16 receptors and 9 ligands for Eph kinases, termed ephrins (19-22). We performed quantitative real-time RT-PCR in SW48 cells to detect the expression of the transcripts encoding EPHA1 and EPHA2 receptors and EFNA1 (ephrin-A1) and EFNA2 (ephrin-A2) ligands. When $N R A S^{+/+}$cells were compared with $N R A S^{Q 61 K /+}$ cells, a trend towards lower expression of the EPHA1 transcript was detected in $N R A S^{Q 61 K /+}$ cells (Fig. 2A).

A completely different picture emerged when the expression of EPHA1, EPHA2, EFNA1, and EFNA2 transcripts was evaluated following 48 -h exposure to cetuximab (Fig. 2B). Whereas the baseline expression of EPHA1 remained unaltered in cetuximab-resistant $N R A S^{Q 61 K /+}$ cells, a significant 1.5 -fold upregulation of the EPHA1 transcript occurred in cetuximab-responsive $N R A S^{+/+}$cells. Furthermore, cetuximab treatment resulted in a robust and significant $>3$-fold downregulation of EPHA2 in $N R A S^{+/+}$cells whereas the expression of EPHA2 remained unaltered in $N R A S^{Q 61 K /+}$ cells. A trend towards higher expression of EFNA1 ligand accompanied the downregulation of EPHA2 in cetuximab-responsive $N R A S^{+/+}$ cells. By contrast, cetuximab treatment failed to change the expression of EFNA1 and EFNA2 ligands in cetuximabresistant $N R A S^{Q 61 K /+}$ cells (Fig. 2C).

Stimulation of ephA2 with a soluble recombinant version of the ephrin-Al ligand restores cetuximab responsiveness in NRAS-
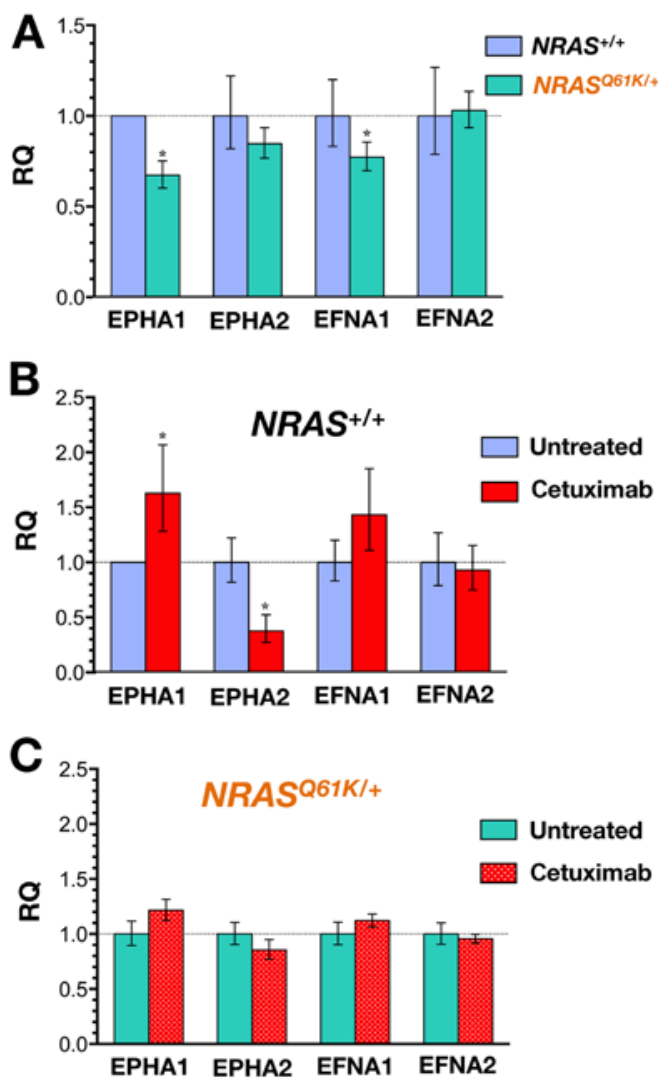

Figure 2. NRAS mutation protects mCRC cells from cetuximab-induced downregulation of EPHA2. Total RNA from $N R A S^{+/+}$and $N R A S^{Q 61 K /+}$ cells cultured in the absence or presence of $100 \mu \mathrm{g} / \mathrm{ml}$ cetuximab $(48 \mathrm{~h})$ was characterized in technical triplicates for the relative abundance of the EPHA1, EPHA2, EFNA1, and EFNA2 mRNAs. The transcript abundance was calculated using the $\Delta \mathrm{Ct}$ method and presented as relative quantification (RQ). 
A
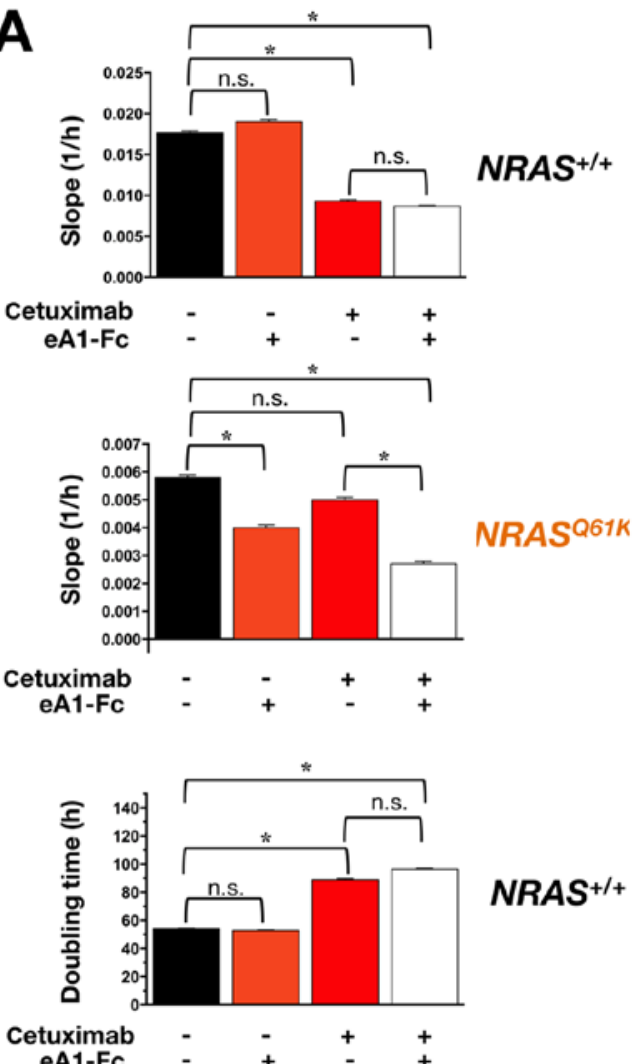

B

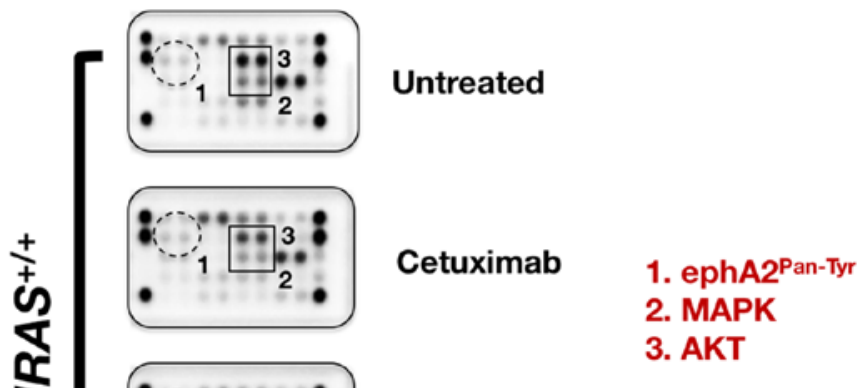

eA1-Fc

-
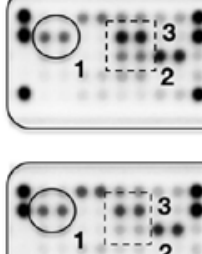

$\bullet$

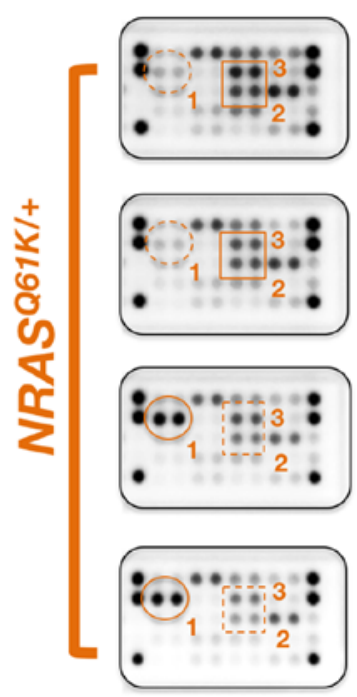

Cetuximab + eA1-Fc

Untreated

Cetuximab

1. ephA2 ${ }^{\text {Pan-Tyr }}$

2. MAPK

3. AKT

eA1-Fc

Cetuximab + eA1-Fc

Figure 3. Pharmacological mimicking of ligand-induced ephA2 signaling restores cetuximab efficacy in NRAS-mutant cells. (A) The rate of proliferation was monitored in real-time using the xCELLligence system. Normalized cell index values obtained in the presence of eA1-Fc $(1 \mu \mathrm{g} / \mathrm{ml}), \mathrm{cetuximab}(100 \mu \mathrm{g} / \mathrm{ml})$, or eA1-Fc + cetuximab as determined by analyzing the growth curves of $N R A S^{+/+}$and $N R A S^{Q 61 K /+}$ cells between 24 and $96 \mathrm{~h}$ are shown. Results are shown as mean (columns) $\pm \mathrm{SD}$ (bars) from at least two experiments in which triplicate wells were analyzed. (B) Representative chemiluminiscent array images from the PathScan Intracellular Signaling array kit showing key phosphorylated signaling nodes in $N R A S^{+/+}$(top panels) and $N R A S^{\text {Q6IK/+ }}$ cell (bottom panels) untreated or treated with cetuximab and/or eA1-Fc are shown. NS, not significant differences. ${ }^{*} \mathrm{P}<0.05$.

mutant cells. Because ligand-independent cross-talk between ephA2 and other oncogenic pathways (e.g., PI3K/AKT and RAS/ERK) results in tumor promotion (19-22), whereas ligand-induced ephA2 signaling triggers intrinsic tumor suppressive signaling involving the blockade of PI3K/AKT and RAS/ERK pathways (21-24), we hypothesized that the ephA2/ephrin-A1 axis might operate as a molecular switch determining the responsiveness/unresponsiveness of cetuximab in NRAS wild-type and NRAS-mutant mCRC cells. To question whether the loss of ligand-dependent signaling changed the function of ephA2 to a 'protector' against cetuximab in NRAS-mutant mCRC cells, we took advantage of the well-documented observation that stimulation of tumor cells with ephrin-A1-Fc (eA1-Fc), a soluble recombinant ephrin-A1 ligand fused to the Fc portion of human immunoglobulin $G$ $(\mathrm{IgG})$, leads to tyrosine phosphorylation of ephA 2 and its downregulation (25-28).
Cell proliferation rates of $\mathrm{NRAS}^{+/+}$and NRAS $S^{Q 61 K /+}$ cells cultured with or without cetuximab, eAl-Fc, or cetuximab plus eAl-Fc were dynamically calculated using impedance technology (Fig. 3A). The cell proliferation rate for NRAS $S^{Q 61 K /+}$ cells treated with cetuximab was significantly higher than that for cetuximab-treated $N R A S^{+/+}$cells, confirming the refractoriness of $N R A S^{Q 61 K /+}$ cells to the anti-proliferative effects of cetuximab. A small reduction in cell proliferation occurred in NRAS $S^{Q 61 K /+}$ cells treated with eA1-Fc but not in $N R A S^{+/+}$cells. The addition of eA1-Fc failed to alter the ability of cetuximab to significantly reduce the proliferation rate of $N R A S^{+/+}$cells. Interestingly, co-treatment with eA1-Fc and cetuximab fully restored the capacity for cetuximab to inhibit the growth of $N R A S^{Q 61 K /+}$ cells.

Stimulation of ephA2 with eA1-Fc suppresses cetuximab-unresponsive hyperactivation of MAPK and AKT in NRAS-mutant cells. To confirm that engagement of the ligand-dependent 

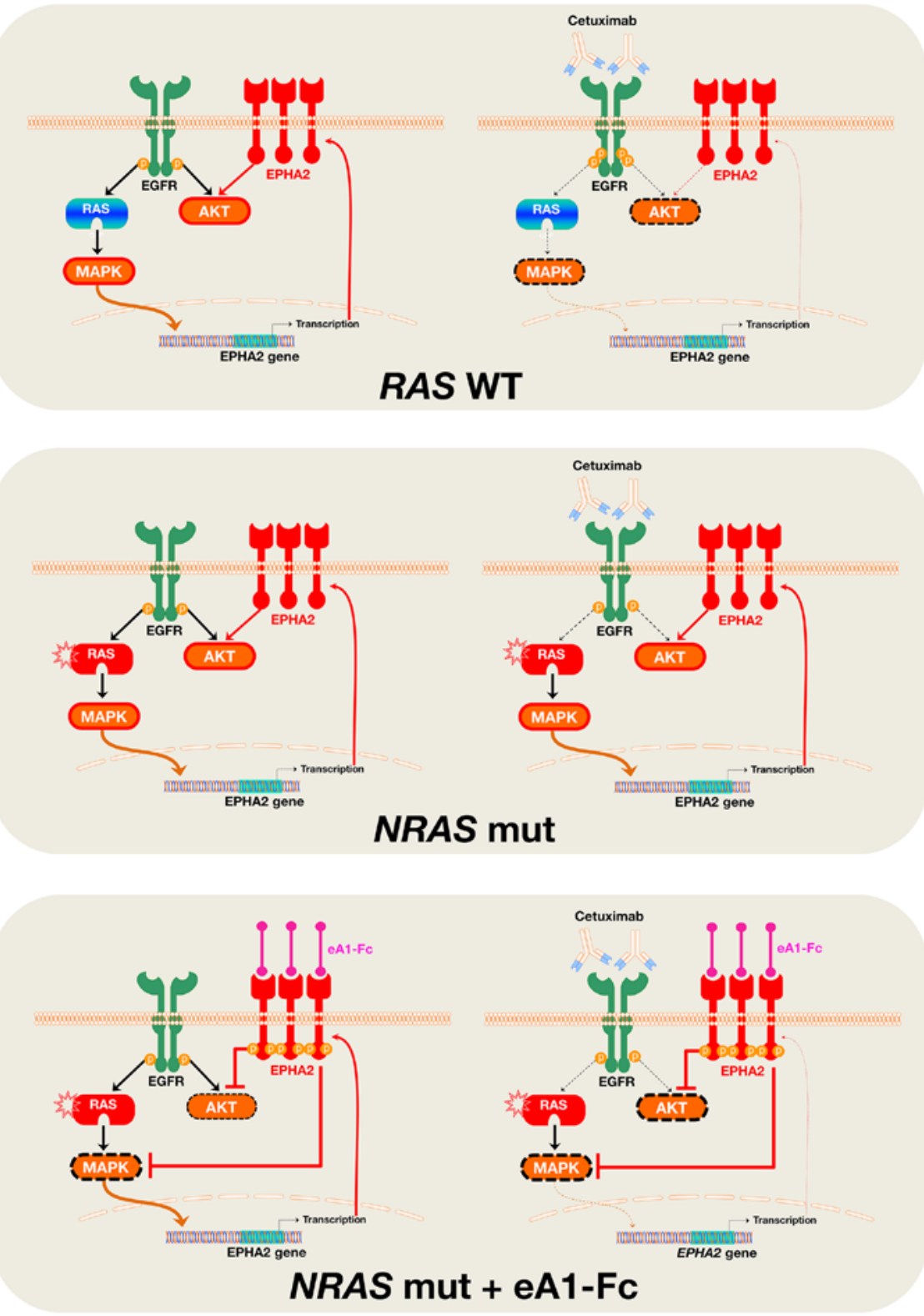

Figure 4. EphA2 signaling and cetuximab responsiveness: a working model. Ligand-independent oncogenic signaling of non-tyrosine phosphorylated ephA2 promotes resistance to cetuximab. In NRAS-mutant cells, EPHA2 gene expression remains constitutively unaltered due to cetuximab-unresponsive activation of MAPK and/or lack of ephrin-A1 ligand-induced ephA2 receptor downregulation. Ligand (ephrin-A1)-induced tyrosine phosphorylated ephA2 signaling restores responsiveness to cetuximab. Upon ephrin-A1 ligation to ephA2, which causes phosphorylation of the tyrosine residues of the receptor and its subsequent downregulation, AKT is dephosphorylated and MAPK is inactivated, thus generating a phospho-phenocopy of cetuximab-sensitive $R A S$ wild-type cells

tumor-suppressive branch of ephA2 signaling synergistically sensitized NRAS-mutant cells to cetuximab via suppression of the pro-oncogenic ligand-independent branch of ephA2 signaling, we used commercially available slide-based antibody arrays to simultaneously assess multiple well-characterized intracellular signaling molecules (Fig. 3B). We confirmed that pharmacological mimicking of ligand-dependent stimulation of ephA2 with eA1-Fc resulted in a significant pan-tyrosination of ephA2. Of note, eA1-Fc-induced activation of ephA2 was stronger in cetuximab-refractory $N R A S^{Q 61 K /+}$ cells than in cetuximab-responsive $N R A S^{+/+}$cells. Co-treatment with eA1-Fc decreased the cetuximab-unresponsive hyperactivation of MAPK in NRAS-mutant cells and decreased also the cetuximab-unresponsive hyperactivation of AKT in NRASmutant cells. Thus, co-treatment with eA1-Fc and cetuximab generates a phospho-signaling signature in NRAS-mutant cells reminiscent to that observed in cetuximab-treated $N R A S^{+/+}$ cells. Indeed, the strong activation of ephA2 with eA1-Fc that occurred in NRAS-mutant cells decreased AKT to levels lower than those observed in $N R A S^{+/+}$cells, where no further changes in the deactivation of MAPK and AKT induced by cetuximab occurred when $N R A S^{+/+}$cells were co-exposed to eA1-Fc (Fig. 3B).

\section{Discussion}

In recent years, the ephrin RTKs and ephrin ligands have been established as integral drivers of cancer formation and progression (19-22). Here we provide evidence that dysregulation of the ephrin-A1/ephA2 signaling axis plays an unexpected role 
in determining the refractoriness of NRAS-mutant mCRC cells to the EGFR-targeted monoclonal antibody cetuximab.

Our findings suggest that suppression of the ligand-independent tumor-promoting signaling of ephA2 might be part of the complex molecular mechanism through which cetuximab exerts its growth inhibitory effects against EGFR-dependent $R A S$ wild-type mCRC cells. Because the ephA2 protein can directly interact with EGFR (29-32), and EPHA2 is a direct transcriptional target of the Ras-Raf-MAPK pathway (33-35), cetuximab-induced blockade of EGFR signaling and subsequent downregulation of MAPK activity leads to a reduction in EPHA2 expression in wild-type RAS mCRC cells. In $N R A S$-mutant mCRC cells, however, the incapacity of cetuximab to block MAPK activity impedes the establishment of the feedback loop that negatively regulates EPHA2 expression (Fig. 4), which ultimately translates into the unresponsiveness of 'NRAS-protected' EPHA2 to the downregulatory effects of cetuximab.

Our claim that cetuximab-directed reduction of EPHA2 expression might be part of the mechanism of action of cetuximab is supported by the finding that function-based targeting of ephA2 signaling was sufficient to fully restore the function of cetuximab in NRAS-mutant mCRC cells. Treatment with eA1-Fc, which generates phenotypes similar to those generated by siRNA-mediated or antisense oligonucleotidemediated genetic knockdown of ephA2 (25-28,36-38), efficiently converted $N R A S$-mutant cells into $R A S$ wild-type cells in terms of cetuximab functioning and efficacy. Upon restoration of the ligand-dependent tumor-suppressive signaling of ephA2 via stimulation with recombinant ephrinA1, the constitutively active MAPK signaling of $N R A S$-mutant mCRC cells was inhibited in the presence of cetuximab. Moreover, supporting and expanding earlier studies attributing ligand-dependent ephA2 activation to suppression of the AKT-mTOR pathway in cancer cells $(23,39)$, treatment with eA1-Fc synergistically interacted with cetuximab to suppress AKT activation in NRAS-mutant cells. Indeed, we observed a significantly stronger phosphorylation of the tyrosine residues of the ephA2 receptor following stimulation with eA1-Fc in $N R A S$-mutant cells (and a more significant decrease in phosphorylation of AKT) than in $R A S$ wild-type cells, strongly suggesting that NRAS-mutant mCRC cells constitutively exhibit an accelerated phosphorylation/dephosphorylation cross-talk between ephA2 and AKT.

Our findings are in line and expand on recent studies demonstrating the involvement of ephA2 in the resistance to small molecule EGFR tyrosine kinase inhibitors, such as erlotinib and gefitinib, in lung cancer $(32,40)$, vemurafenib in melanoma (41), and the anti-HER2 monoclonal antibody trastuzumab in breast cancer (42-44). It might be argued that the strength of evidence provided by the sole isogenic cell line pair $\left(N R A S^{+/+}\right.$ vs $\left.N R A S^{Q 61 K /+}\right)$ used in our current approach precludes any general extrapolation to mCRC patients. However, it should be noted that, while elucidating new molecular processes contributing to CRC pathogenesis using ephA $2^{\text {high }}$-sorted cell subpopulations with stem-like features purified from a chemically-induced model of sporadic colon carcinogenesis, De Robertis et al recently reported that dysregulated expression of the ephA2 receptor accompanied by downregulation of the ligand EFNA1 might operate as a novel mechanism of resistance to cetuximab that can be considered an alternative to KRAS mutations (45). We conclude that, even in the absence of constitutive overexpression of ephA2 in NRAS-mutant cells, dysregulated signaling of the ephrin-A1/ephA2 axis suffices to overcome the inhibition of EGFR signaling imposed by cetuximab. Future studies should examine whether the altered functioning of the ephrin-A1/ephA2 axis might confer stemlike properties to $N R A S$-mutant mCRC cells, thus explaining the shortened survival and lack of response to anti-EGFR treatment of NRAS-mutant mCRC patients (1-9).

Because both ephA2 and EFNA1 are recognized as novel biomarkers of benefit from cetuximab-based therapy in mCRC independently of the KRAS mutation status (45-47), our current findings might help to delineate the ephrin-A1/ ephA2 signaling axis as a common mechanism of cetuximab resistance involving all $\mathrm{mCRC}$ patients. Moreover, the fact that cetuximab functioning apparently involves also the upregulation of ephA1, whose reduced expression correlates with poor differentiation, invasion, metastasis and poor overall survival in CRC (48), further underscores the unappreciated relevance of ephrin receptors and ephrin ligands in the clinico-molecular management of mCRC.

In conclusion, our results reveal that: a) the clinical benefit of cetuximab in mCRC might involve the suppression of the ligandless oncogenic state of the eph $\mathrm{A} 2$ receptor; b) imparting ligand-dependent tumor suppressing signaling through ephA2 restores the responsiveness of NRAS-mutant mCRC cells to cetuximab. The fact that $N R A S$-mutant mCRC cells molecularly behave like $R A S$ wild-type cells upon ephrin-A1 signaling to ephA2, in terms of cetuximab efficacy, might open new therapeutic opportunities to clinically widen the usage of cetuximab in mCRC patients.

\section{Acknowledgements}

This study was supported by grants from the Ministerio de Ciencia e Innovación (Grant SAF2016-80639-P), Plan Nacional de I+D+I, Spain and the Agència de Gestió d'Ajuts Universitaris i de Recerca (AGAUR) (grant 2014 SGR229), Departament d'Economia I Coneixement, Catalonia, Spain, to Javier A. Menendez. Bernardo Queralt and Javier A. Menendez thank a charity collection organized by Fundació Roses Contra el Càncer (Roses, Girona, Catalonia) that allowed this line of research to be initiated in 2014. The Metabolism and Cancer Laboratory is supported by an unrestricted grant from the Joan Armangué family (Girona, Catalonia). The authors would like to thank Dr Kenneth McCreath for editorial support.

\section{References}

1. De Roock W, Claes B, Bernasconi D, De Schutter J, Biesmans B, Fountzilas G, Kalogeras KT, Kotoula V, Papamichael D, Laurent-Puig P, et al: Effects of KRAS, BRAF, NRAS, and PIK3CA mutations on the efficacy of cetuximab plus chemotherapy in chemotherapy-refractory metastatic colorectal cancer: A retrospective consortium analysis. Lancet Oncol 11: 753-762, 2010.

2. Peeters M, Oliner KS, Parker A, Siena S, Van Cutsem E, Huang J, Humblet Y, Van Laethem JL, André T, Wiezorek J, et al: Massively parallel tumor multigene sequencing to evaluate response to panitumumab in a randomized phase III study of metastatic colorectal cancer. Clin Cancer Res 19: 1902-1912, 2013. 
3. Misale S, Di Nicolantonio F, Sartore-Bianchi A, Siena S and Bardelli A: Resistance to anti-EGFR therapy in colorectal cancer: From heterogeneity to convergent evolution. Cancer Discov 4: 1269-1280, 2014.

4. Meriggi F, Vermi W, Bertocchi P and Zaniboni A: The emerging role of NRAS mutations in colorectal cancer patients selected for anti-EGFR therapies. Rev Recent Clin Trials 9: 8-12, 2014.

5. Bronte G, Silvestris N, Castiglia M, Galvano A, Passiglia F, Sortino G, Cicero G, Rolfo C, Peeters M, Bazan V, et al: New findings on primary and acquired resistance to anti-EGFR therapy in metastatic colorectal cancer: Do all roads lead to RAS? Oncotarget 6: 24780-24796, 2015.

6. Hecht JR, Douillard JY, Schwartzberg L, Grothey A, Kopetz S, Rong A, Oliner KS and Sidhu R: Extended RAS analysis for anti-epidermal growth factor therapy in patients with metastatic colorectal cancer. Cancer Treat Rev 41: 653-659, 2015.

7. Schirripa M, Cremolini C, Loupakis F, Morvillo M, Bergamo F, Zoratto F, Salvatore L, Antoniotti C, Marmorino F, Sensi E, et al: Role of NRAS mutations as prognostic and predictive markers in metastatic colorectal cancer. Int J Cancer 136: 83-90, 2015.

8. Hsu HC, Thiam TK, Lu YJ, Yeh CY, Tsai WS, You JF, Hung HY, Tsai CN, Hsu A, Chen HC, et al: Mutations of KRAS/NRAS/ BRAF predict cetuximab resistance in metastatic colorectal cancer patients. Oncotarget 7: 22257-22270, 2016

9. Taniguchi H, Yamazaki K, Yoshino T, Muro K, Yatabe Y, Watanabe T, Ebi H, Ochiai A, Baba E and Tsuchihara K; Japanese Society of Medical Oncology: Japanese Society of Medical Oncology Clinical Guidelines: RAS (KRAS/NRAS) mutation testing in colorectal cancer patients. Cancer Sci 106: 324-327, 2015.

10. Allegra CJ, Rumble RB, Hamilton SR, Mangu PB, Roach N, Hantel A and Schilsky RL: Extended RAS gene mutation testing in metastatic colorectal carcinoma to predict response to antiepidermal growth factor receptor monoclonal antibody therapy: American Society of Clinical Oncology Provisional Clinical Opinion Update 2015. J Clin Oncol 34: 179-185, 2016.

11. Irahara N, Baba Y, Nosho K, Shima K, Yan L, Dias-Santagata D, Iafrate AJ, Fuchs CS, Haigis KM and Ogino S: NRAS mutations are rare in colorectal cancer. Diagn Mol Pathol 19: 157-163, 2010.

12. Janakiraman M, Vakiani E, Zeng Z, Pratilas CA, Taylor BS, Chitale D, Halilovic E, Wilson M, Huberman K, Ricarte Filho JC et al: Genomic and biological characterization of exon 4 KRAS mutations in human cancer. Cancer Res 70: 5901-5911, 2010.

13. Haigis KM, Kendall KR, Wang Y, Cheung A, Haigis MC Glickman JN, Niwa-Kawakita M, Sweet-Cordero A, SeboltLeopold J, Shannon KM, et al: Differential effects of oncogenic $\mathrm{K}-\mathrm{Ras}$ and N-Ras on proliferation, differentiation and tumor progression in the colon. Nat Genet 40: 600-608, 2008.

14. Wang Y, Velho S, Vakiani E, Peng S, Bass AJ, Chu GC, Gierut J, Bugni JM, Der CJ, Philips M, et al: Mutant N-RAS protects colorectal cancer cells from stress-induced apoptosis and contributes to cancer development and progression. Cancer Discov 3: 294-307, 2013.

15. Queralt $B$, Cuyàs E, Bosch-Barrera J, Massaguer A, de Llorens R, Martin-Castillo B, Brunet J, Salazar R and Menendez JA: Synthetic lethal interaction of cetuximab with MEK1/2 inhibition in NRAS-mutant metastatic colorectal cancer. Oncotarget 7 : 82185-82199, 2016.

16. Mandic R, Rodgarkia-Dara CJ, Zhu L, Folz BJ, Bette M, Weihe E, Neubauer A and Werner JA: Treatment of HNSCC cell lines with the EGFR-specific inhibitor cetuximab (Erbitux) results in paradox phosphorylation of tyrosine 1173 in the receptor. FEBS Lett 580: 4793-4800, 2006.

17. Yoshida T, Okamoto I, Okabe T, Iwasa T, Satoh T, Nishio K Fukuoka M and Nakagawa K: Matuzumab and cetuximab activate the epidermal growth factor receptor but fail to trigger downstream signaling by Akt or Erk. Int J Cancer 122: 1530-1538, 2008

18. Oliveras-Ferraros C, Vazquez-Martin A,López-Bonet E, MartínCastillo B, Del Barco S, Brunet J and Menendez JA: Growth and molecular interactions of the anti-EGFR antibody cetuximab and the DNA cross-linking agent cisplatin in gefitinib-resistan MDA-MB-468 cells: New prospects in the treatment of triplenegative/basal-like breast cancer. Int J Oncol 33: 1165-1176, 2008.

19. Pasquale EB: Eph-ephrin bidirectional signaling in physiology and disease. Cell 133: 38-52, 2008

20. Pasquale EB: Eph receptors and ephrins in cancer: Bidirectional signalling and beyond. Nat Rev Cancer 10: 165-180, 2010
21. Wykosky J and Debinski W: The EphA2 receptor and ephrinA1 ligand in solid tumors: Function and therapeutic targeting. Mol Cancer Res 6: 1795-1806, 2008.

22. Beauchamp A and Debinski W: Ephs and ephrins in cancer: ephrin-A1 signalling. Semin Cell Dev Biol 23: 109-115, 2012

23. Miao H, Li DQ, Mukherjee A, Guo H, Petty A, Cutter J, Basilion JP, Sedor J, Wu J, Danielpour D, et al: EphA2 mediates ligand-dependent inhibition and ligand-independent promotion of cell migration and invasion via a reciprocal regulatory loop with Akt. Cancer Cell 16: 9-20, 2009.

24. Petty A, Myshkin E, Qin H, Guo H, Miao H, Tochtrop GP, Hsieh JT, Page P, Liu L, Lindner DJ, et al: A small molecule agonist of EphA2 receptor tyrosine kinase inhibits tumor cell migration in vitro and prostate cancer metastasis in vivo. PLoS One 7: e42120, 2012

25. Carter N, Nakamoto T, Hirai H and Hunter T: EphrinA1-induced cytoskeletal re-organization requires FAK and p130(cas). Nat Cell Biol 4: 565-573, 2002.

26. Duxbury MS, Ito H, Zinner MJ, Ashley SW and Whang EE: Ligation of EphA2 by Ephrin A1-Fc inhibits pancreatic adenocarcinoma cellular invasiveness. Biochem Biophys Res Commun 320: 1096-1102, 2004.

27. Wykosky J, Gibo DM, Stanton C and Debinski W: EphA2 as a novel molecular marker and target in glioblastoma multiforme. Mol Cancer Res 3: 541-551, 2005.

28. Walker-Daniels J, Riese DJ II and Kinch MS: c-Cbl-dependent EphA2 protein degradation is induced by ligand binding. Mol Cancer Res 1: 79-87, 2002.

29. Ramnarain DB, Park S, Lee DY, Hatanpaa KJ, Scoggin SO, Otu H, Libermann TA, Raisanen JM, Ashfaq R, Wong ET, et al: Differential gene expression analysis reveals generation of an autocrine loop by a mutant epidermal growth factor receptor in glioma cells. Cancer Res 66: 867-874, 2006.

30. Larsen AB, Pedersen MW, Stockhausen MT, Grandal MV, van Deurs B and Poulsen HS: Activation of the EGFR gene target EphA2 inhibits epidermal growth factor-induced cancer cell motility. Mol Cancer Res 5: 283-293, 2007.

31. Larsen AB, Stockhausen MT and Poulsen HS: Cell adhesion and EGFR activation regulate EphA2 expression in cancer. Cell Signal 22: 636-644, 2010

32. Koch H, Busto ME, Kramer K, Médard G and Kuster B: Chemical proteomics uncovers EPHA2 as a mechanism of acquired resistance to small molecule EGFR kinase inhibition. J Proteome Res 14: 2617-2625, 2015

33. Miao H, Wei BR, Peehl DM, Li Q, Alexandrou T, Schelling JR, Rhim JS, Sedor JR, Burnett E and Wang B: Activation of EphA receptor tyrosine kinase inhibits the Ras/MAPK pathway. Nat Cell Biol 3: 527-530, 2001.

34. Macrae M, Neve RM, Rodriguez-Viciana P, Haqq C, Yeh J, Chen C, Gray JW and McCormick F: A conditional feedback loop regulates Ras activity through EphA2. Cancer Cell 8: 111-118, 2005.

35. Pratt RL and Kinch MS: Ligand binding up-regulates EphA2 messenger RNA through the mitogen-activated protein/extracellular signal-regulated kinase pathway. Mol Cancer Res 1: 1070-1076, 2003

36. Liu F, Park PJ, Lai W, Maher E, Chakravarti A, Durso L, Jiang X, Yu Y, Brosius A, Thomas M, et al: A genome-wide screen reveals functional gene clusters in the cancer genome and identifies EphA2 as a mitogen in glioblastoma. Cancer Res 66 10815-10823, 2006.

37. Carles-Kinch K, Kilpatrick KE, Stewart JC and Kinch MS: Antibody targeting of the EphA2 tyrosine kinase inhibits malignant cell behavior. Cancer Res 62: 2840-2847, 2002.

38. Shao H, Pandey A, O'Shea KS, Seldin M and Dixit VM: Characterization of $\mathrm{B} 61$, the ligand for the Eck receptor proteintyrosine kinase. J Biol Chem 270: 5636-5641, 1995.

39. Miao H, Gale NW, Guo H, Qian J, Petty A, Kaspar J, Murphy AJ, Valenzuela DM, Yancopoulos G, Hambardzumyan D, et al: EphA2 promotes infiltrative invasion of glioma stem cells in vivo through cross-talk with Akt and regulates stem cell properties. Oncogene 34: 558-567, 2015.

40. Amato KR, Wang S, Tan L, Hastings AK, Song W, Lovly CM, Meador CB, Ye F, Lu P, Balko JM, et al: EPHA2 blockade overcomes acquired resistance to EGFR kinase inhibitors in lung cancer. Cancer Res 76: 305-318, 2016.

41. Miao B, Ji Z, Tan L, Taylor M, Zhang J, Choi HG, Frederick DT, Kumar R, Wargo JA, Flaherty KT, et al: EPHA2 is a mediator of vemurafenib resistance and a novel therapeutic target in melanoma. Cancer Discov 5: 274-287, 2015 
42. Zhuang G, Brantley-Sieders DM, Vaught D, Yu J, Xie L, Wells S, Jackson D, Muraoka-Cook R, Arteaga C and Chen J: Elevation of receptor tyrosine kinase EphA2 mediates resistance to trastuzumab therapy. Cancer Res 70: 299-308, 2010.

43. Menyhárt O, Santarpia L and Győrffy B: A comprehensive outline of trastuzumab resistance biomarkers in HER2 overexpressing breast cancer. Curr Cancer Drug Targets 15: 665-683, 2015.

44. Youngblood VM, Kim LC, Edwards DN, Hwang Y, Santapuram PR, Stirdivant SM, Lu P, Ye F, Brantley-Sieders DM and Chen J: The ephrin-A1/EPHA2 signaling axis regulates glutamine metabolism in HER2-positive breast cancer. Cancer Res 76: 1825-1836, 2016.

45. De Robertis M, Loiacono L, Fusilli C, Poeta ML, Mazza T, Sanchez M, Marchionni L, Signori E, Lamorte G, Vescovi AL, et al: Dysregulation of EGFR pathway in EphA2 cell subpopulation significantly associates with poor prognosis in colorectal cancer. Clin Cancer Res 23: 159-170, 2017.
46. Pentheroudakis G, Kotoula V, De Roock W, Kouvatseas G, Papakostas P, Makatsoris T, Papamichael D, Xanthakis I, Sgouros J, Televantou D, et al: Biomarkers of benefit from cetuximab-based therapy in metastatic colorectal cancer: Interaction of EGFR ligand expression with RAS/RAF, PIK3CA genotypes. BMC Cancer 13: 49, 2013.

47. Strimpakos A, Pentheroudakis G, Kotoula V, De Roock W, Kouvatseas G, Papakostas P, Makatsoris T, Papamichael D, Andreadou A, Sgouros J, et al: The prognostic role of ephrin A2 and endothelial growth factor receptor pathway mediators in patients with advanced colorectal cancer treated with cetuximab. Clin Colorectal Cancer 12: 267-274.e2, 2013.

48. Dong Y, Wang J, Sheng Z, Li G, Ma H, Wang X, Zhang R, Lu G, $\mathrm{Hu} \mathrm{Q}$, Sugimura H, et al: Downregulation of EphA1 in colorectal carcinomas correlates with invasion and metastasis. Mod Pathol 22: 151-160, 2009. 